\title{
The Neutral Line Concept in Hot-Rolled Steel Cross-Sections
}

\author{
Ioannis G. Raftoyiannis and George T. Michaltsos* \\ Department of Civil Engineering, National Technical University of Athens, Greece
}

\begin{abstract}
The present work deals with the effect of the thermally-induced residual stresses on the shape of a crosssection's neutral line for hot-rolled steel beams under combined loading conditions. In general, the neutral line of a beam cross-section is considered to be straight. In this study it is shown that, taking into account the existing thermal residual stresses, this line becomes a curve as far as the beam is subjected to pure bending or to combined loading that consists of a bending moment and an axial force.
\end{abstract}

Keywords: Steel sections, Hot-rolled sections, Neutral line, Thermal stresses, Residual stresses.

\section{INTRODUCTION}

The lifetime of a structural member is usually determined by the interaction between the defects within the member and the stress states to which it is subjected to, regardless of its material properties.

Contrarily to the externally applied loads, which can be readily and comprehensively introduced in the design of structural members, it is considerably more difficult to account for residual stresses in both the analysis and the design procedure.

This is true, since residual stresses vary significantly in magnitude, they are strongly non-linear (in distribution and nature), unpredictable and cannot be reliably measured.

Focusing on hot-rolled steel structural elements, the most common and simultaneously unavoidable type of residual stresses arising are the so called process-induced ones, which can be either flow-induced or thermally-induced, with the latter being the dominant component.

The mechanism of the development of thermally-induced residual stresses and their distribution for steel structural elements can be found in detail in numerous text-books, papers and reports (Huber and Beedle [1], Alpsten [2], Michaltsos [3], and others not quoted herein).

For the majority of widely used hot-rolled beams with sections as I-sections or orthogonal ones, this distribution is symmetric along the primary axes and thus the effect of such a self-equilibrated stress system is neglected either in elastic analysis or in elastoplastic one, mainly when simple (pure) loading conditions are specified, i.e. when the distribution of the stresses due to external loads is also symmetric.

However, this is not the case when combined loads are acting upon the component, as for beam-columns, moment

*Address correspondence to this author at the Department of Civil Engineering National Technical University of Athens, 9 Iroon Polytechneiou Str, Zografou Campus, Athens, 15780 Greece; Tel: +30-210-7722482; Fax:+30-210-7723443; E-mail: michalts@central.ntua.gr frame beams, crane structures etc. Shifting of the neutral axis may cause a significant reduction of the moment resistance and subsequently of the overall bearing capacity of the member. With the continuing encouragement not only to optimize material performance, but simultaneously to minimize component weight, the primary advantages of steel (as for example ductility) must be utilized to the maximum extent.

In doing so, elastoplastic or plastic analysis ought to be used in the design of structural steel-work, and in order to provide reliable results, the effect of thermal residual stresses must be accounted for.

The existence of efficient non-destructive or semidestructive techniques for measuring to a considerable depth the aforementioned stresses (e.g. neutral diffraction or crack compliance respectively), offers to engineering practice the capacity of a quantitative ascertainment of the amount of thermal residual stresses on a wide range of hot-rolled steel sections.

To the knowledge of the authors, however, there seems to be a lack of practice design guidelines and tools, based on solid theoretical background for taking into account the aforementioned stresses.

A limited number of publications exist in the relevant literature, but it deals mostly with built-up or complex structural components, as in Marcelin [4] or Dixit and Dixit [5]. The effect of thermal residual stresses on bearing capacity of hot rolled sections under either combined bending moment and axial force or, also, combined bending moment and shearing force is already proven $[6,7]$.

The present work aims to contribute to the specific topic mentioned above, and especially to deal with the effect of thermal-induced residual stresses on the form of the neutral line of hot-rolled sections under the action of bending moment and axial force. With the use of the elementary elastoplastic analysis, simple formulae are obtained and characteristic examples are presented. 


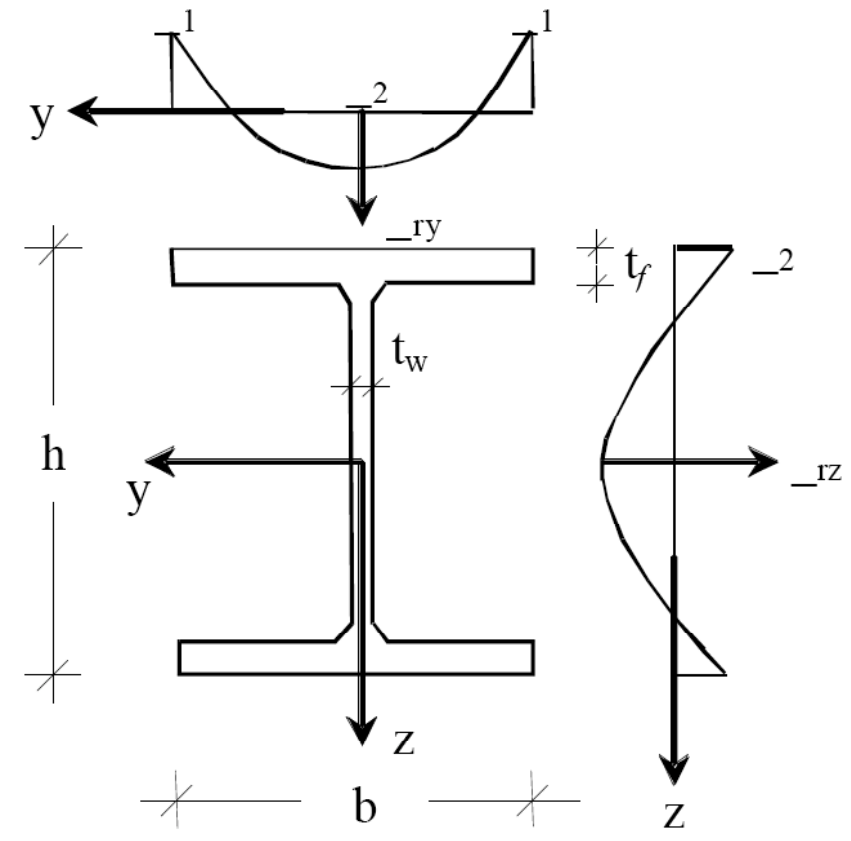

Fig. (1a). Residual stresses in a steel I-section.

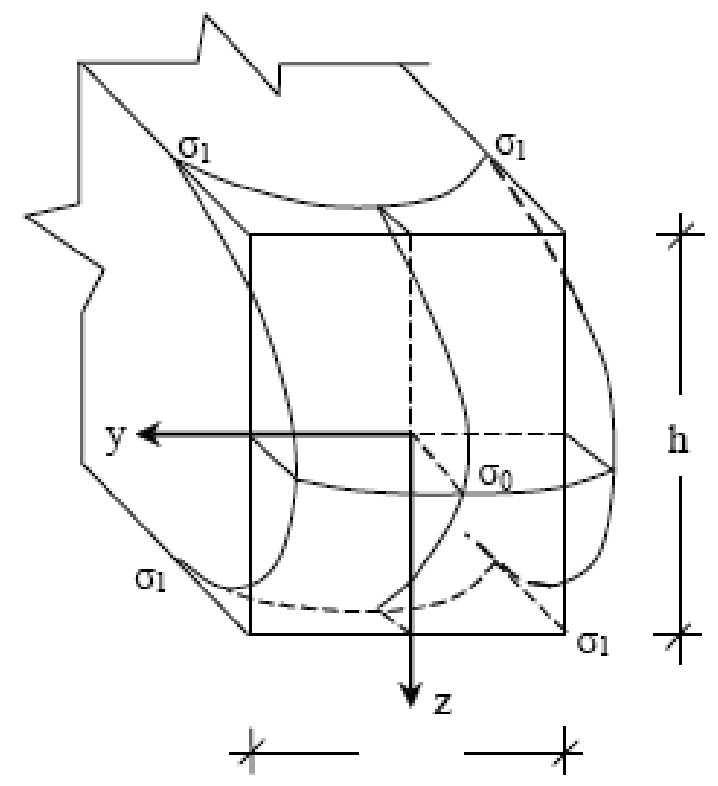

Fig. (1b). Residual stresses in an orthogonal steel cross-section.

\section{EFFECT OF THERMALLY-INDUCED RESIDUAL STRESSES}

The residual stresses are due to the way of treatment and production of the structural steel and they constitute a selfequilibrated stress state. Therefore, they are usually ignored in the analysis.

The distribution and magnitude of the thermal residual stresses can be readily taken form Alpsten [2] as well as from Eurocode 3 [8], and are shown in Fig. (1a) for a steel Isection and in Fig. (1b) for a solid orthogonal cross-section.

\section{The I-Section}

For the distribution shown in Fig. (1a), and taking into account the conditions:

$$
\begin{aligned}
& \sigma_{r y}(0)=\sigma_{2}, \quad \sigma_{r y}\left( \pm \frac{b}{2}\right)=\sigma_{1}, \quad \sigma_{r y}(0)=\sigma_{r z}\left( \pm \frac{h}{2}\right) \\
& 2 t_{f} \int_{-b / 2}^{b / 2} \sigma_{r y}(y) d y+t_{w} \int_{-h / 2}^{h / 2} \sigma_{r z}(z) d z=0
\end{aligned}
$$

one obtains

$$
\begin{aligned}
& \sigma_{r y}(y)=\frac{4\left(\sigma_{1}-\sigma_{2}\right)}{b^{2}} \cdot y^{2}+\sigma_{2} \\
& \sigma_{r z}(z)=A \cdot z^{2}+B
\end{aligned}
$$

where

$$
\begin{aligned}
& A=\frac{4 A_{f}\left(\sigma_{1}+2 \sigma_{2}\right)+3 \sigma_{2}\left(A_{w}-t_{f} t_{w}\right)}{t_{w}\left(2 h^{3}-3 h t_{f}^{2}+t_{f}^{3}\right)} \\
& B=\sigma_{2}-\frac{h^{2}}{4} A \\
& A_{f}=2 b t_{f}, \quad A_{w} \cong t_{w} h
\end{aligned}
$$

\section{The Solid Orthogonal Cross-Section}

For the distribution showing in Fig. (1b), and taking into account the conditions:

$$
\begin{aligned}
& \sigma_{r}\left( \pm \frac{b}{2}, \pm \frac{h}{2}\right)=\sigma_{1}, \quad \sigma_{r}(0,0)=\sigma_{o} \\
& \int_{-b / 2}^{b / 2} d y \int_{-h / 2}^{h / 2} \sigma_{r}(y, z) d z=0
\end{aligned}
$$

one can find

$$
\sigma_{r}(y, z)=A\left(y^{2}+z^{2}\right)+B y^{2} z^{2}+\Gamma
$$

where

$$
\begin{aligned}
& A=-\frac{2\left(\sigma_{1}+8 \sigma_{o}\right)}{b^{2}+h^{2}} \\
& B=\frac{24\left(\sigma_{1}+2 \sigma_{o}\right)}{b^{2} h^{2}} \\
& \Gamma=\sigma_{o}
\end{aligned}
$$

As it is shown in the followings, the thermally-induced residual stresses cause a displacement of the neutral line of the cross-section in Fig. (1a), while for the one in Fig. (1b) the neutral line changes to a curve as well.

\section{EXTERNAL LOADS AND RESIDUAL STRESSES}

Let us consider now, a cross-section subjected to the action of a moment $\mathrm{M}_{\mathrm{y}}$ and an axial force $\mathrm{N}_{\mathrm{x}}$.

As the above moment and axial force increase, the crosssection is strained and enters from the elastic region firstly into elastoplastic region and then into the plastic one - see Figs. (2a) to (2c).

It has been proved that the full plasticization of a crosssection cannot be realized from a theoretical point of view. A small elastic kernel always remains near the neutral line (in its final position) that behaves elastically, since any more 
a) Elastic region

a)

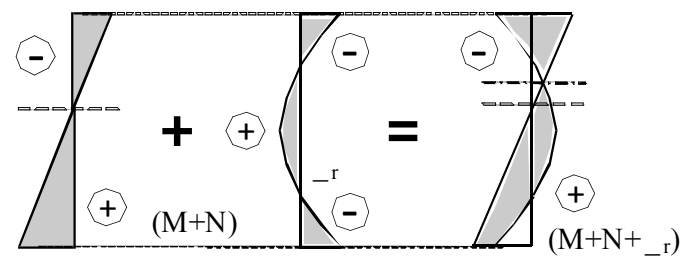

b) Elastoplastic regio $n$

b)

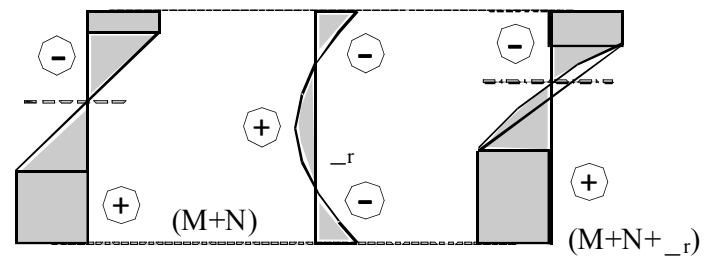

c) Plastic region

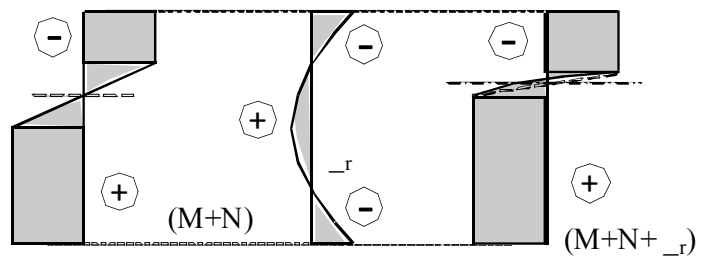

d)

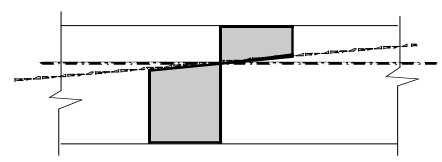

Fig. (2). Elastic - plastic zones due to bending.

plasticization involves an extremely large (theoretically infinite) deformation of the external fibers, which in fact cannot take place - see Fig. (2d).

This elastic kernel extends from $2 \%$ to $4 \%$ of the total cross-section area. Therefore, a situation like the one shown in Fig. (2c) will be considered as a fully plasticized crosssection.

\section{THE ELASTIC REGION}

\section{The I-Section}

Ignoring the thermal residual stresses, the position of the neutral line is given by the expression:
$z_{o}=-\frac{I_{b} \cdot N_{x}}{A_{b} \cdot M_{y}}$

where $M_{y}$ and $N_{x}$ are the moment and the axial force and $I_{b}$ and $A_{b}$ are the cross-section's moment of inertia and area, respectively.

Taking into account the thermal residual stresses, we have:

$\sigma_{x}=\frac{M_{y}}{I_{b}} z+\frac{N_{x}}{A_{b}}+\sigma_{r z}$

For the determination of the neutral line position will be: $\sigma_{\mathrm{x}}=0$, or because of Eq. (1)

$\frac{M_{y}}{I_{b}} z_{n}+\frac{N_{x}}{A_{b}}+A \cdot z_{n}^{2}+B=0$

and finally:

$$
z_{n}=\frac{1}{2 \cdot A} \cdot\left(-\frac{M_{y}}{I_{b}}+\sqrt{\left(\frac{M_{y}}{I_{b}}\right)^{2}-4 \cdot A \cdot\left(B+\frac{N_{x}}{A_{b}}\right)}\right)
$$

In Fig. (3) one can see the old $\left(\mathrm{z}_{\mathrm{o}}\right)$ and the new position $\left(z_{n}\right)$ of the neutral line as well as the corresponding diagrams of $\sigma_{\mathrm{x}}$ for the case of a bending moment acting alone and of a simultaneous action of a bending moment and an axial force.

\section{The Solid Orthogonal Cross-Section}

Ignoring the thermal residual stresses, the position of the neutral axis is given also by the expression (3), while taking into accent the thermal residual stresses we get the expression (4). By setting $\sigma_{x}=0$ and because of Eq.(2) we obtain:

$\frac{M_{y}}{I_{b}} z_{n}+\frac{N_{x}}{A_{b}}+A \cdot\left(y^{2}+z_{n}^{2}\right)+B y^{2} z^{2}+\Gamma=0$

which finally concludes to the following expression:

$$
\begin{aligned}
z_{n} & =\frac{1}{2 \cdot\left(A+B \cdot y^{2}\right)} \cdot\left(-\frac{M_{y}}{I_{b}}+\right. \\
& \left.+\sqrt{\left(\frac{M_{y}}{I_{b}}\right)^{2}-4 \cdot\left(A+B \cdot y^{2}\right) \cdot\left(A \cdot y^{2}+\Gamma+\frac{N_{x}}{A_{b}}\right)}\right)
\end{aligned}
$$

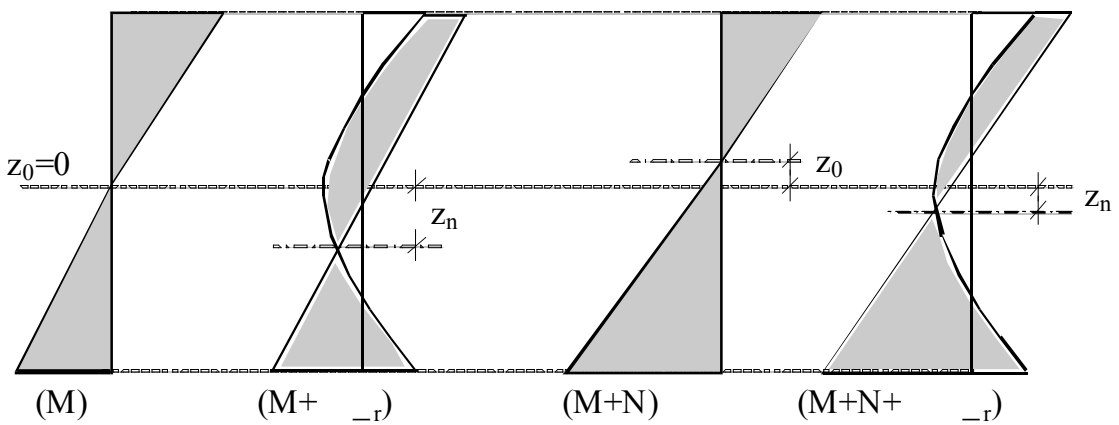

Fig. (3). Stress distribution diagrams. 


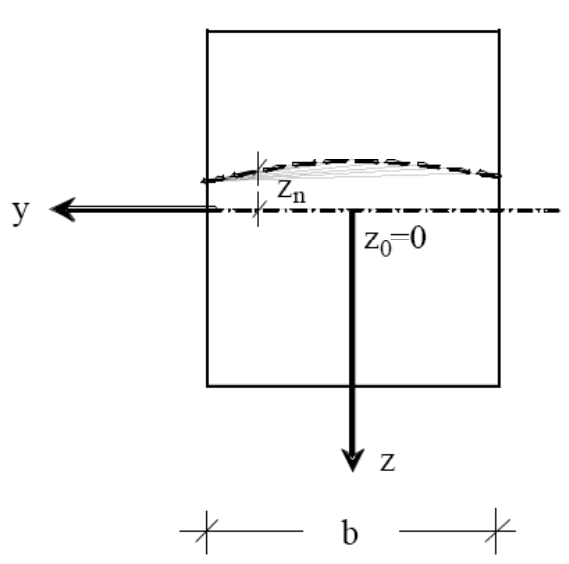

a) $\left(\mathrm{M}+{ }_{-}\right)$

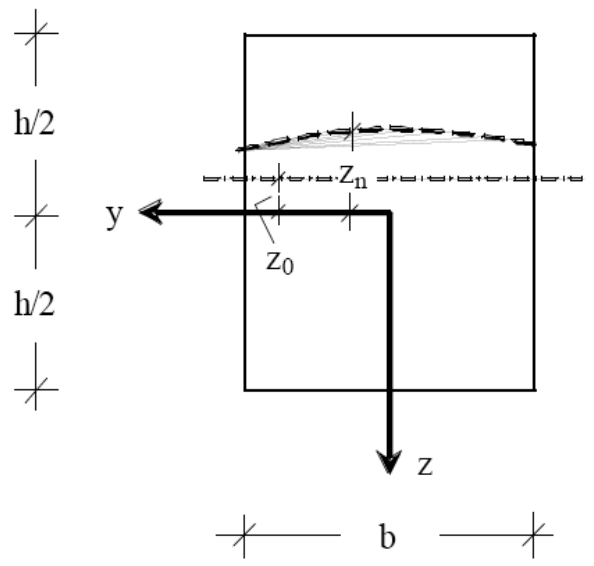

b) $\left(\mathrm{M}+\mathrm{N}+{ }_{\mathrm{r}}\right)$

Fig. (4). Neutral line positions due to bending, axial and thermal stresses.
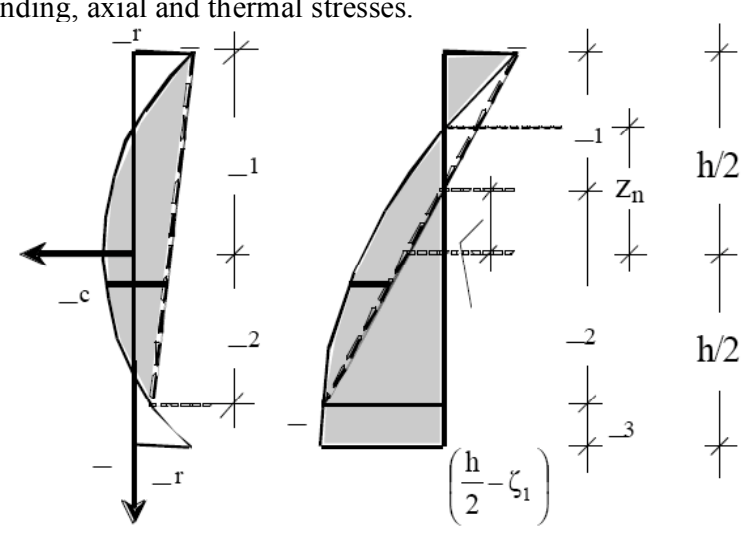

Fig. (5a). Neutral line position with plastic region at the bottom.

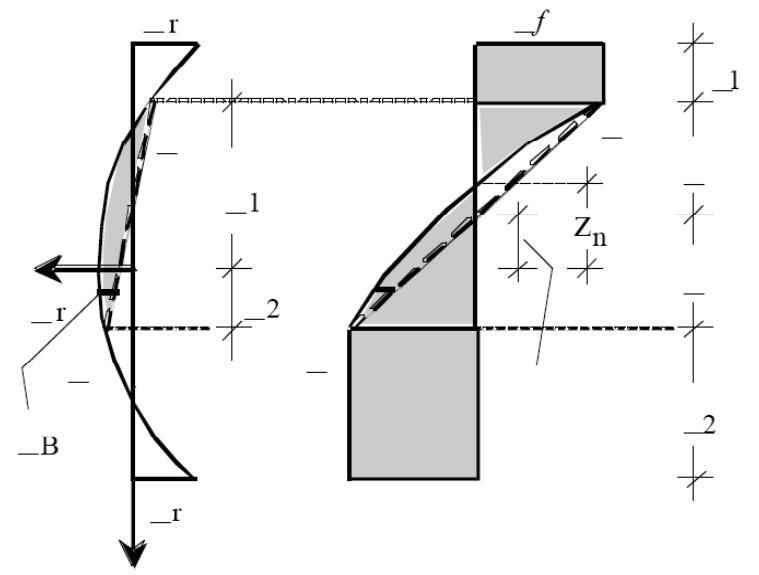

Fig. (5b). Neutral line position with top and bottom plastic regions.

Fig. (4) shows the new position of the neutral line for the case of a pure moment (Fig. 4a) and of a simultaneous action of a moment and an axial force (Fig. 4b).

We discern that, except the change of its position, the neutral line changes from a straight line into a curve.

\section{ELASTOPLASTIC AND PLASTIC REGIONS}

As the influence of the thermal residual stresses is more evident and indicative on solid orthogonal cross-sections, the following analysis focuses on this type of cross-sections.
Once a cross-section enters into the elastoplastic region, the distribution of stresses (including the thermal residual ones) will have one of the forms shown in Fig. (5a) or Fig. (5b) - see also Fig. (2).

The part $\Gamma \Delta$ of diagrams (5a) and (5b) is a curve having the same form with the part $\mathrm{AB}$ of the $\sigma_{\mathrm{r}}$ - diagram. This curve may be determined as follows:

$$
\begin{array}{ll}
\text { Po int } A: & \sigma_{r A}=A \cdot\left(y^{2}+\alpha_{1}^{2}\right)+B \cdot y^{2} \alpha_{1}^{2}+\Gamma \\
\text { Po int } B: & \sigma_{r B}=A \cdot\left(y^{2}+\alpha_{2}^{2}\right)+B \cdot y^{2} \alpha_{2}^{2}+\Gamma
\end{array}
$$




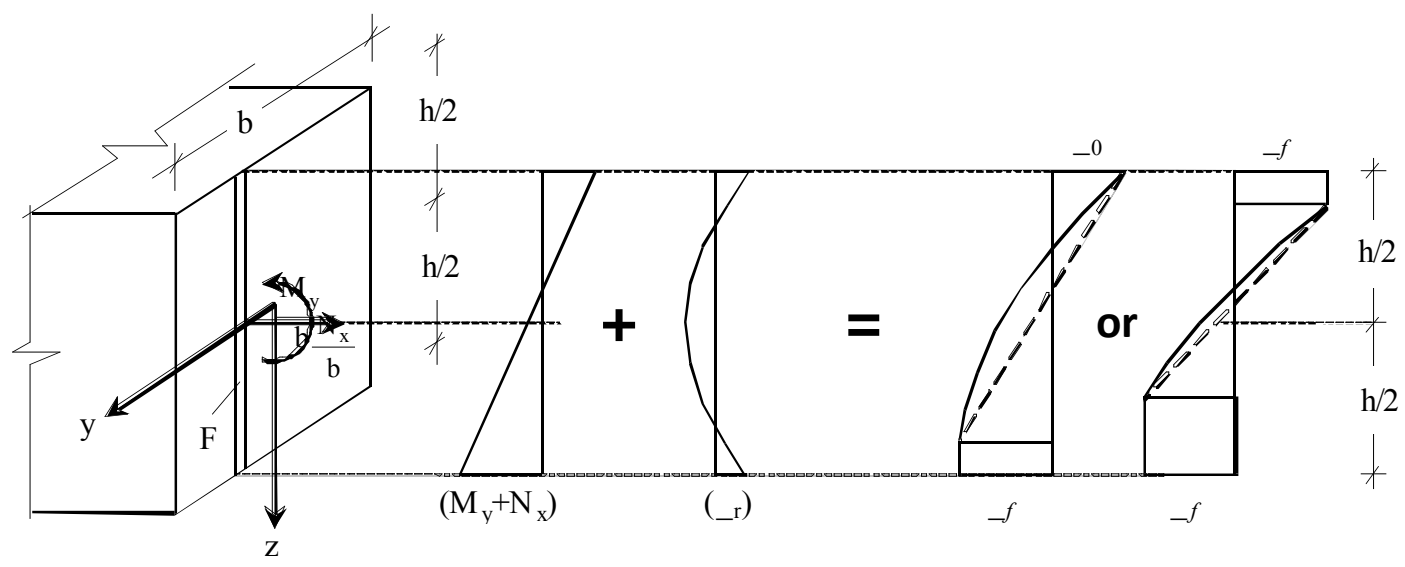

Fig. (6). Stress distribution in a solid cross-section.

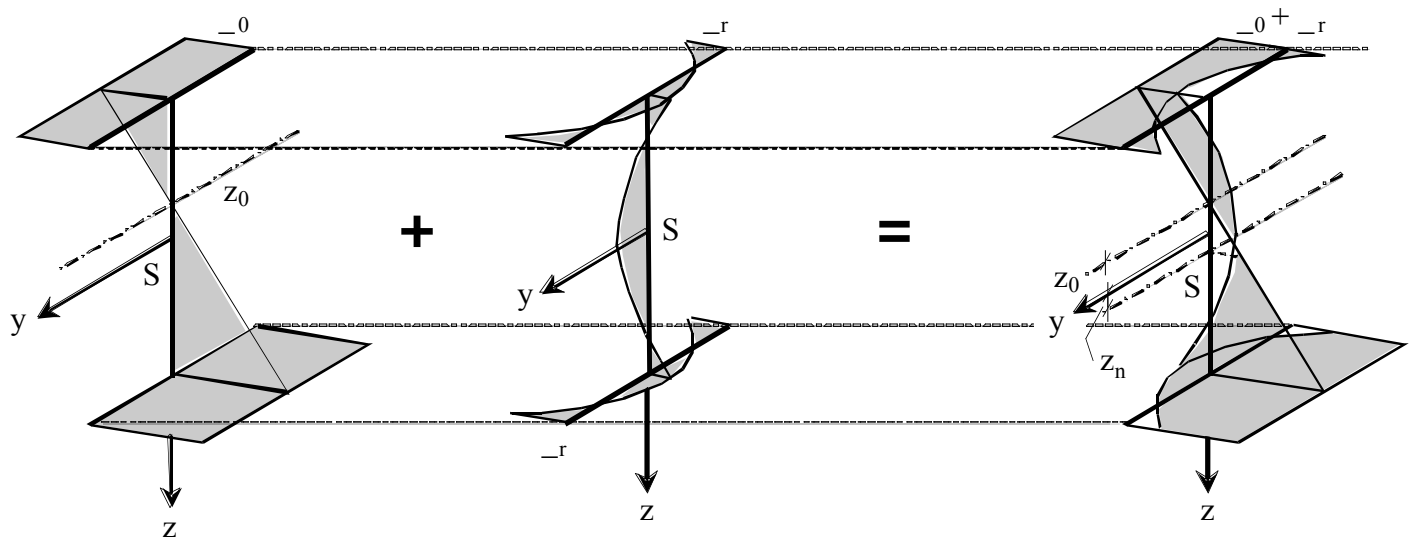

Fig. (7). Stress distribution in an I-section.

The equation of the straight line $\mathrm{AB}$ is:

$\frac{\sigma_{A B}-\sigma_{r A}}{\sigma_{r A}-\sigma_{r B}}=\frac{z-\alpha_{1}}{\alpha_{1}-\alpha_{2}}$ or

$\sigma_{A B}=\frac{z-\alpha_{1}}{\alpha_{1}-\alpha_{2}}\left(\sigma_{r A}-\sigma_{r B}\right)+\sigma_{r A}=$

$=\frac{z-\alpha_{1}}{\alpha_{1}-\alpha_{2}}\left(\alpha_{1}^{2}-\alpha_{2}^{2}\right)\left(A+B y^{2}\right)+A\left(y^{2}+\alpha_{1}^{2}\right)+B y^{2} \alpha_{1}^{2}+\Gamma$

or finally:

$$
\begin{array}{r}
\sigma_{A B}=\left(z-\alpha_{1}\right)\left(\alpha_{1}+\alpha_{2}\right)\left(A+B y^{2}\right)+ \\
+A\left(y^{2}+\alpha_{1}^{2}\right)+B y^{2} \alpha_{1}^{2}+\Gamma
\end{array}
$$

and the $\sigma_{\mathrm{c}}$ (from the hatched section of diagram $\sigma_{\mathrm{r}}$ ) is given by the relation:

$$
\begin{aligned}
\mathrm{o}_{\mathrm{c}}= & \mathrm{o}_{\mathrm{r}}-\mathrm{o}_{\mathrm{AB}}= \\
= & \mathrm{A}\left(\mathrm{y}^{2}+\mathrm{z}^{2}\right)+\mathrm{By}^{2} \mathrm{z}^{2}+\mathrm{A}-\left(\mathrm{z}-\mathrm{a}_{1}\right)\left(\dot{a}_{1}+\mathrm{a}_{2}\right)\left(\mathrm{A}+\mathrm{By}^{2}\right)- \\
& -\mathrm{A}\left(\mathrm{y}^{2}+\mathrm{a}_{1}^{2}\right)-\mathrm{By}^{2} \mathrm{a}_{1}^{2}-\mathrm{A}= \\
= & \mathrm{A}\left(\mathrm{z}^{2}-\alpha_{1}^{2}\right)+B y^{2}\left(\mathrm{z}^{2}-\alpha_{1}^{2}\right)-\left(\mathrm{z}-\mathrm{a}_{1}\right)\left(\mathrm{a}_{1}+\mathrm{a}_{2}\right)\left(\mathrm{A}+\mathrm{By}{ }^{2}\right)
\end{aligned}
$$

or finally:

$$
\sigma_{c}=\left(z-\alpha_{1}\right)\left(z-\alpha_{2}\right)\left(A+B y^{2}\right)
$$

The case in Fig. (5a)

With the notations of Fig. (5a), we have:

$$
\begin{aligned}
& \alpha_{1}=\frac{h}{2}, \quad \alpha_{2}=\zeta_{1}+\zeta_{2}-\frac{h}{2}, \\
& \zeta_{3}=h-\zeta_{1}-\zeta_{2}, \quad \sigma_{o}=\sigma_{f} \frac{\zeta_{1}}{\zeta_{2}}
\end{aligned}
$$

therefore, the unknown $\zeta_{1}$ and $\zeta_{2}$ can be determined through the following conditions:

$\int_{-h / 2}^{h / 2} \sigma d z=\frac{N_{x}}{b}$ and $\int_{-h / 2}^{h / 2} \sigma z d z=\frac{M_{y}}{b}$

The first condition gives:

$\frac{N_{x}}{b}=\sigma_{f}\left(h-\zeta_{1}-\zeta_{2}\right)+\frac{1}{2} \sigma_{f} \zeta_{2}-\frac{1}{2} \sigma_{f} \zeta_{1}+\int_{\alpha_{1}}^{\alpha_{2}} \sigma_{c} d z$

or finally:

$\sigma_{f}\left(h-\zeta_{1}-\frac{\zeta_{2}}{2}-\frac{\zeta_{1}^{2}}{2 \zeta_{2}}\right)+\frac{\left(\alpha_{1}-\alpha_{2}\right)^{3}}{6}\left(A+B y^{2}\right)=\frac{N_{x}}{b}$

The second condition gives:

$$
\begin{aligned}
\frac{M_{y}}{b}=\frac{1}{2} \sigma_{o} \zeta_{1}\left(\frac{h}{2}-\right. & \left.\frac{\zeta_{1}}{3}\right)+\frac{1}{2} \sigma_{f} \zeta_{2}\left(\frac{2 \zeta_{2}}{3}-\frac{h}{2}+\zeta_{1}\right)+ \\
& +\sigma_{f} \zeta_{3}\left(\frac{h}{2}-\frac{\zeta_{3}}{2}\right)+\int_{\alpha_{1}}^{\alpha_{2}} \sigma_{c}\left(z+\frac{h}{2}-\zeta_{1}\right) d z
\end{aligned}
$$

or finally: 
$\sigma_{f}\left[\frac{\zeta_{1}^{2}}{2 \zeta_{2}}\left(\frac{h}{2}-\frac{\zeta_{1}}{3}\right)+\frac{\zeta_{2}}{2}\left(\frac{2 \zeta_{2}}{3}+\zeta_{1}-\frac{h}{2}\right)+\zeta_{3}\left(\frac{h}{2}-\frac{\zeta_{3}}{2}\right)\right]+$

$+\frac{\left(\alpha_{1}+\alpha_{2}\right)\left(\alpha_{1}-\alpha_{2}\right)^{3}}{12}\left(A+B y^{2}\right)+$

$+\left(\frac{h}{2}-\zeta_{1}\right) \frac{\left(\alpha_{1}-\alpha_{2}\right)^{3}}{6}\left(A+B y^{2}\right)=\frac{M_{y}}{b}$

Equations (12) and (13) constitute a system, the solution of which gives the unknowns $\zeta_{1}$ and $\zeta_{2}$.

In order to define the position of the neutral line, we have to determine the equation of the curve $\Gamma \Delta$ (see Fig. 5a).

The equation of the straight line $\overline{\Gamma \Delta}$ is given by the relation:

$\frac{\sigma_{\Gamma \Delta}-\sigma_{o}}{\sigma_{o}-\sigma_{f}}=\frac{z-\alpha_{1}}{\alpha_{1}-\alpha_{2}}$

or

$\sigma_{\Gamma \Delta}=\frac{z-\alpha_{1}}{\alpha_{1}-\alpha_{2}}\left(\sigma_{o}-\sigma_{f}\right)+\sigma_{o}$

and the equation of the curve $\Gamma \Delta$ is:

$$
\begin{aligned}
\sigma= & \sigma_{\Gamma \Delta}+\sigma_{c}=\frac{z-\alpha_{1}}{\alpha_{1}-\alpha_{2}}\left(\sigma_{o}-\sigma_{f}\right)+ \\
& +\sigma_{o}+\left(z-\alpha_{1}\right)\left(z-\alpha_{2}\right)\left(A+B y^{2}\right)
\end{aligned}
$$

with the notations of Eq.(10).

The solution of the equation $\sigma=0$ gives the neutral line $z_{n}$ for various values of $y$.

The case in Fig. (5b)

With the notations of Fig. (5b) we have:

$\zeta=\frac{\alpha_{1}+\alpha_{2}}{2}, \quad \zeta_{1}=\frac{h}{2}-\alpha_{1}$,

$\zeta_{2}=\frac{h}{2}-\alpha_{2}, \quad \zeta_{1}-\zeta_{2}=\alpha_{1}-\alpha_{2}$

Where, the unknowns $\alpha_{1}$ and $\alpha_{2}$, can be determined by the conditions (11).

The first condition gives:

$\frac{N_{x}}{b}=\sigma_{f}\left(\zeta_{2}-\zeta_{1}\right)+\int_{\alpha_{1}}^{\alpha_{2}} \sigma_{c} d z$

or finally:

$\sigma_{f}\left(\zeta_{2}-\zeta_{1}\right)+\frac{\left(\alpha_{1}-\alpha_{2}\right)^{3}}{6}\left(A+B y^{2}\right)=\frac{N_{x}}{b}$

The second condition gives:

$$
\begin{aligned}
& \frac{M_{y}}{b}=\sigma_{f} \zeta_{1}\left(\alpha_{1}+\frac{\zeta_{1}}{2}\right)+\sigma_{f} \zeta_{2}\left(\alpha_{2}+\frac{\zeta_{2}}{2}\right)+ \\
& +\sigma_{f} \frac{2 \zeta^{2}}{3}+\int_{\alpha_{1}}^{\alpha_{2}} \sigma_{c}\left(z+\frac{h}{2}-\zeta-\zeta_{1}\right) d z
\end{aligned}
$$

which concludes to:

$$
\begin{aligned}
& \sigma_{f}\left[\alpha_{1} \zeta_{1}+\alpha_{2} \zeta_{2}+\frac{\zeta_{1}^{2}+\zeta_{2}^{2}}{2}+\frac{2 \zeta^{2}}{3}\right]+ \\
& +\frac{\left(\alpha_{1}+\alpha_{2}\right)\left(\alpha 1-\alpha_{2}\right)^{3}}{12}\left(A+B y^{2}\right)+ \\
& +\left(\frac{h}{2}-\zeta-\zeta_{1}\right) \frac{\left(\alpha 1-\alpha_{2}\right)^{3}}{6}\left(A+B y^{2}\right)=\frac{M_{y}}{b}
\end{aligned}
$$

Equations (16) and (17) constitute a system, the solution of which gives the unknowns $\alpha_{1}$ and $\alpha_{2}$.

The equation of the straight line $\overline{\Gamma \Delta}$ is given by the relation:

$\frac{\sigma_{\Gamma \Delta}+\sigma_{f}}{-\sigma_{f}-\sigma_{f}}=\frac{z-\alpha_{1}}{\alpha_{1}-\alpha_{2}}$

or

$\sigma_{\Gamma \Delta}=-2 \cdot \frac{z-\alpha_{1}}{\alpha_{1}-\alpha_{2}} \sigma_{f}-\sigma_{f}$

and the equation that gives the curve $\Gamma \Delta$ is:

$$
\begin{aligned}
\sigma=\sigma_{\Gamma \Delta} & +\sigma_{c}=\frac{2\left(z-\alpha_{1}\right)}{\alpha_{1}-\alpha_{2}} \sigma_{f}-\sigma_{f}+ \\
& +\left(z-\alpha_{1}\right)\left(z-\alpha_{2}\right)\left(A+B y^{2}\right)
\end{aligned}
$$

The solution of the equation $\sigma=0$ gives again the neutral line $z_{n}$ for various values of $y$ (See also Fig. (6)).

\section{NUMERICAL RESULTS AND DISCUSSION}

\section{The Elastic Region}

Let us consider an IPE200 standard steel profile with $\sigma_{f}=$ $3000 \mathrm{dN} / \mathrm{cm}^{2}$ which has the following properties: $h=20 \mathrm{~cm}$, $\mathrm{b}=10 \mathrm{~cm}, t_{f}=0.85 \mathrm{~cm}, t_{w}=0.56 \mathrm{~cm}, \mathrm{I}_{\mathrm{y}}=1940 \mathrm{~cm}^{4}, A_{b}=$ $38.50 \mathrm{~cm}^{2}, A_{w}=10.36 \mathrm{~cm}^{2}$. We also assume that the thermal residual stresses have the form shown in Fig. (1a) with values $\sigma_{1}=-400 d N / \mathrm{cm}^{2}, \sigma_{2}=400 d N / \mathrm{cm}^{2}$.

The cross-section is subjected to the moment $M_{y}=200000$ $\mathrm{dNcm}$ and an axial force $\mathrm{N}_{\mathrm{x}}=5000 \mathrm{dN}$.

Ignoring at first the thermal residual stresses, the neutral line is found at:

$$
z_{o}=-\frac{N_{x} / A_{b}}{M_{y} / I_{y}}=-\frac{5000 \cdot 1940}{200000 \cdot 38.50}=-1.259 \mathrm{~cm}
$$

while the stresses $\sigma_{\mathrm{o}}$ and $\sigma_{\mathrm{u}}$ at the upper and lower fibers, respectively, are:

$$
\begin{aligned}
\sigma_{o, u}=\frac{M_{y}}{I_{y}} & \cdot\left(\mp \frac{h}{2}\right)+\frac{N_{x}}{A_{b}}= \\
= & \mp \frac{200000}{1940} \cdot 10+\frac{5000}{38.50}=\left\{\begin{array}{l}
-901.05 \mathrm{dN} / \mathrm{cm}^{2} \\
1160.79 \mathrm{l}
\end{array}\right.
\end{aligned}
$$

Applying Eq.(5), we obtain: 


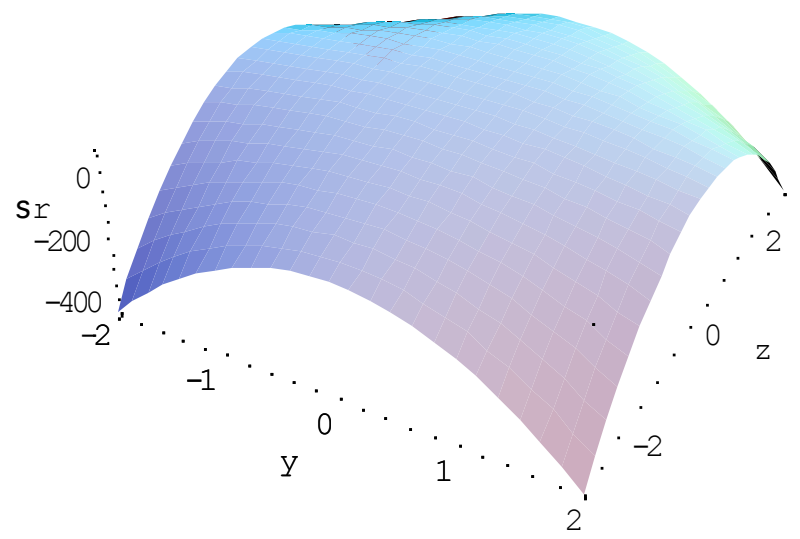

Fig. (8). Thermal stresses in a solid cross-section.

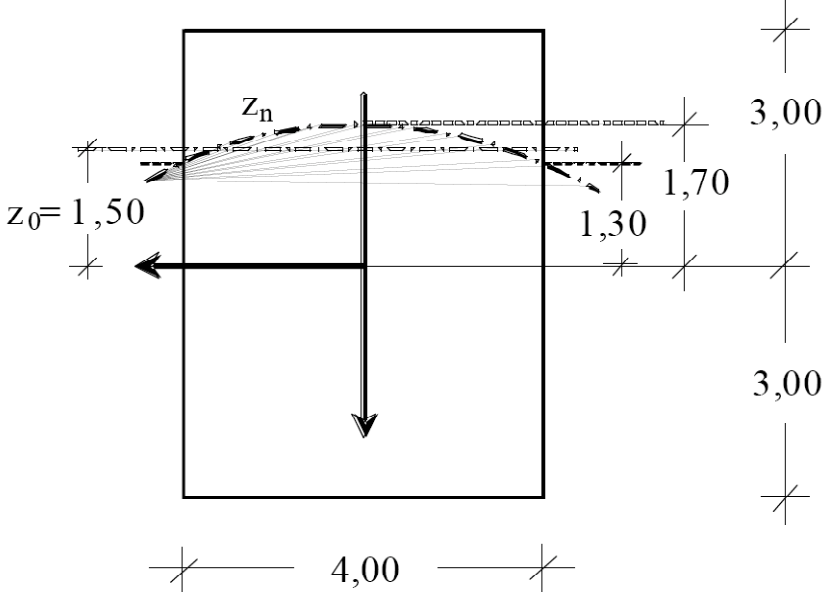

Fig. (9). Displaced position of the neutral line.

$z_{n}=+2.54 \mathrm{~cm}$

Let us see now the influence of the thermal residual stresses on the neutral line and the resistant moments. Ignoring the thermal residual stresses, we have:

$W_{o}=\frac{1940}{10-1.259}=221.94 \mathrm{~cm}^{3}$

$W_{u}=\frac{1940}{10+1.259}=172.31 \mathrm{~cm}^{3}$

Taking into account the influence of the thermal residual stresses we have:

$W_{o}=\frac{1940}{10+2.54}=154.70 \mathrm{~cm}^{3}$

$W_{u}=\frac{1940}{10-2.54}=260.05 \mathrm{~cm}^{3}$

We consider next an orthogonal solid cross-section of dimensions $b^{*} h=4 \times 6 \mathrm{~cm}^{2}$, and we consider in addition that the residual stresses have the form shown in Fig. (8) with

$\sigma_{1}=-400 \mathrm{dN} / \mathrm{cm}^{2}$ and $\sigma_{o}=100 \mathrm{dN} / \mathrm{cm}^{2}$.

For the above cross-section we have: $I_{y}=72 \mathrm{~cm}^{4}, A_{b}=24 \mathrm{~cm}^{2}$.

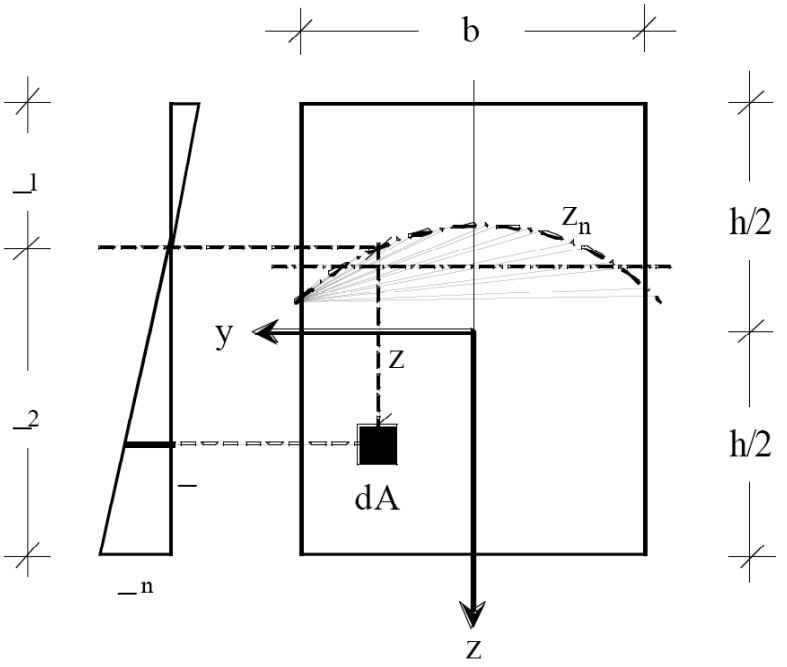

Fig. (10). The influence of thermal stresses in the form of neutral line.

Under the action of a moment $\mathrm{M}_{\mathrm{y}}=24000 \mathrm{dNcm}$ and an axial force $N_{x}=12000 d N$, we find that the neutral line $z_{0}$ (without the influence of the thermal residual stresses) will be at the position:

$z_{o}=-\frac{N_{x} / A_{b}}{M_{y} / I_{y}}=-\frac{12000 \cdot 72}{24000 \cdot 24}=-1.50 \mathrm{~cm}$

Applying the Eq. (6), we find the curve of the neutral line $z_{n}$ as it is shown in Fig. (9), where the neutral axis $z_{o}$ (without the influence of the thermal residual stresses) is also shown.

Let us see now the influence of the thermal residual stresses on the neutral line and the resistant moments. Without the influence of $\sigma_{\mathrm{r}}$ we have:

$$
\begin{aligned}
& M=\int_{A} \sigma \cdot z d A=\int_{A_{1}} \sigma_{o} \frac{z}{\zeta_{1}} z d A_{1}+\int_{A_{2}} \sigma_{u} \frac{z}{\zeta_{2}} z d A_{2}= \\
& =b \sigma_{u}\left(\int_{0}^{\zeta_{1}} \frac{z^{2}}{\zeta_{2}} d z+\int_{0}^{\zeta_{2}} \frac{z^{2}}{\zeta_{2}} d z\right)=b \sigma_{u} \frac{\zeta_{1}^{2}+\zeta_{2}^{2}}{3 \zeta_{2}}
\end{aligned}
$$

and finally we obtain:

$$
W_{u}=b \cdot \frac{\zeta_{1}^{2}+\zeta_{2}^{2}}{3 \zeta_{2}} \text { and } W_{o}=b \cdot \frac{\zeta_{1}^{2}+\zeta_{2}^{2}}{3 \zeta_{1}}
$$

Taking into account the influence of $\sigma_{\mathrm{r}}$ and through a similar process, we obtain:

$\mathrm{W}_{\mathrm{u}}=\frac{1}{3} \int_{-\mathrm{b} / 2}^{\mathrm{b} / 2} \frac{\zeta_{1}^{2}+\zeta_{2}^{2}}{\zeta_{2}} \mathrm{dy}$ and $\mathrm{W}_{\mathrm{o}}=\frac{1}{3} \int_{-\mathrm{b} / 2}^{\mathrm{b} / 2} \frac{\zeta_{1}^{2}+\zeta_{2}^{2}}{\zeta_{1}} \mathrm{dy}$

From the above equations, without the influence of the thermal residual stresses we have: $\mathrm{W}_{\mathrm{o}}=84 \mathrm{~cm}^{3}$ and $\mathrm{W}_{\mathrm{u}}=28$ $\mathrm{cm}^{3}$, while with the influence of the thermal residual stresses we have: $\mathrm{W}_{\mathrm{o}}=93.17 \mathrm{~cm}^{3}$ and $\mathrm{W}_{\mathrm{u}}=28.75 \mathrm{~cm}^{3}$ (See also Fig. (10)).

\section{The Elasto-Plastic and Plastic Regions}

Considering again the orthogonal solid cross-section, one can easily determine the plastic moment (the moment which 


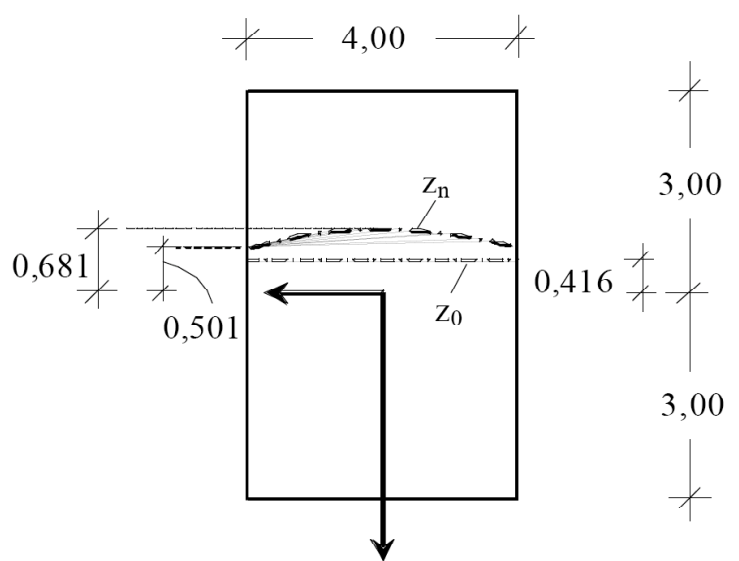

Fig. (11). Neutral line evolving to curve.

causes full plastification of the cross-section): $\mathrm{M}_{\mathrm{F}}=108000$ dNcm.

Under the action of a moment $\mathrm{M}_{\mathrm{y}}=80000 \mathrm{dNcm}$ and an axial force $\mathrm{N}_{\mathrm{x}}=10000 \mathrm{dN}$, and ignoring the stresses $\sigma_{\mathrm{r}}$, the neutral axis will be at $\mathrm{z}_{\mathrm{O}}=-0.4167 \mathrm{~cm}$.

Considering the stress distribution $\sigma_{\mathrm{r}}$ shown in Fig. (7) and applying Eqs. (16), (17) and (18), we find that the neutral line becomes a curve as shown in Fig. (11).

Ignoring the influence of $\sigma_{\mathrm{r}}$, we have:

$$
\begin{aligned}
& M=\int_{A} \sigma z d A=2 \int_{A_{\varepsilon}} \sigma_{f} \frac{z^{2}}{\zeta} d A_{\varepsilon}+ \\
& +b \sigma_{f}\left(\zeta_{1} \frac{h_{1}+\zeta}{2}+\zeta_{2} \frac{h_{2}+\zeta}{2}\right)
\end{aligned}
$$

and since it is: $\zeta_{1}=\mathrm{h}_{1}-\zeta$, and $\zeta_{2}=\mathrm{h}_{2}-\zeta$ (see Fig. 12a,b), we get:

$$
\begin{aligned}
& M=\left.2 b \sigma_{f} \frac{z^{3}}{3 \zeta}\right|_{0} ^{\zeta}+ \\
& +b \sigma_{f}\left(\frac{\left(h_{1}+\zeta\right)\left(h_{1}-\zeta\right)}{2}+\frac{\left(h_{2}+\zeta\right)\left(h_{2}-\zeta\right)}{2}\right)= \\
& =\sigma_{f} b\left(\frac{h_{1}^{2}+h_{2}^{2}}{2}-\frac{\zeta^{2}}{3}\right)
\end{aligned}
$$

and: $W=\frac{b}{2}\left(h_{1}^{2}+h_{2}^{2}\right)-\frac{b \zeta^{2}}{3}$

Taking into account the influence of $\sigma_{\mathrm{r}}$ it is valid that: $\sigma=\sigma_{\mathrm{f}} \frac{z}{\zeta}$

and, therefore:

$$
\begin{aligned}
M & =\int_{A} \sigma \bar{z} d A= \\
& =2 \int_{A_{\varepsilon}} \sigma_{f} \frac{\bar{z}^{2}}{\zeta} d A_{\varepsilon}+\sigma_{f} \int_{-b / 2}^{b / 2} \zeta_{1} \frac{h_{1}+\zeta}{2} d y+ \\
& +\sigma_{f} \int_{-b / 2}^{b / 2} \zeta_{2} \frac{h_{2}+\zeta}{2} d y+\int_{-b / 2}^{b / 2} d y \int_{\alpha_{1}}^{\alpha_{2}} \sigma_{c}\left(z+\frac{h}{2}-h_{1}\right) d z
\end{aligned}
$$

and since it is: $\zeta_{1}=\mathrm{h}_{1}-\zeta$ and $\zeta_{2}=\mathrm{h}_{2}-\zeta$, we obtain:

$$
\begin{aligned}
& M=\sigma_{f} \int_{-b / 2}^{b / 2}\left(\frac{h_{1}^{2}+h_{2}^{2}}{2}-\frac{\zeta^{3}}{3}\right) d y+ \\
& +\int_{-b / 2}^{b / 2} \frac{\left(\alpha_{1}+\alpha_{2}\right)\left(\alpha_{1}-\alpha_{2}\right)^{3}}{12}\left(A+B y^{2}\right) d y+ \\
& +\int_{-b / 2}^{b / 2}\left(\frac{h}{2}-h_{1}\right) \frac{\left(\alpha_{1}-\alpha_{2}\right)^{3}}{6}\left(A+B y^{2}\right) d y= \\
& =\sigma_{f} \int_{-b / 2}^{b / 2}\left(\frac{h_{1}^{2}+h_{2}^{2}}{2}-\frac{\zeta^{3}}{3}+\frac{\left(\alpha_{1}-\alpha_{2}\right)^{3}}{12}\left(\zeta_{1}+\zeta_{2}-2 h_{1}\right) \frac{A+B y^{2}}{\sigma_{f}}\right) d y
\end{aligned}
$$

or finally:

$$
\left.W=\int_{-b / 2}^{b / 2}\left(\begin{array}{r}
\frac{h_{1}^{2}+h_{2}^{2}}{2}-\frac{\zeta^{3}}{3}+\frac{\left(\alpha_{1}-\alpha_{2}\right)^{3}}{12}\left(\zeta_{1}+\zeta_{2}-\right. \\
\left.-2 h_{1}\right)\left(\frac{2 k+16 \lambda}{b^{2}+h^{2}}+\frac{24 k+48 \lambda}{b^{2} h^{2}} y^{2}\right.
\end{array}\right)\right) d y
$$

where: $k=\frac{\sigma_{1}}{\sigma_{f}}$ and $\lambda=\frac{\sigma_{o}}{\sigma_{f}}$

From the above expressions, without the influence of $\sigma_{\mathrm{r}}$ we get: $\mathrm{W}=28.0556 \mathrm{~cm}^{3}$ while with the influence of $\sigma_{\mathrm{r}}$ we get: $\mathrm{W}=23.7684 \mathrm{~cm}^{3}$.

\section{CONCLUSIONS}

This paper studies the influence of the distribution of the thermal residual stresses on the position as well as the form of the neutral line through a simple approach.

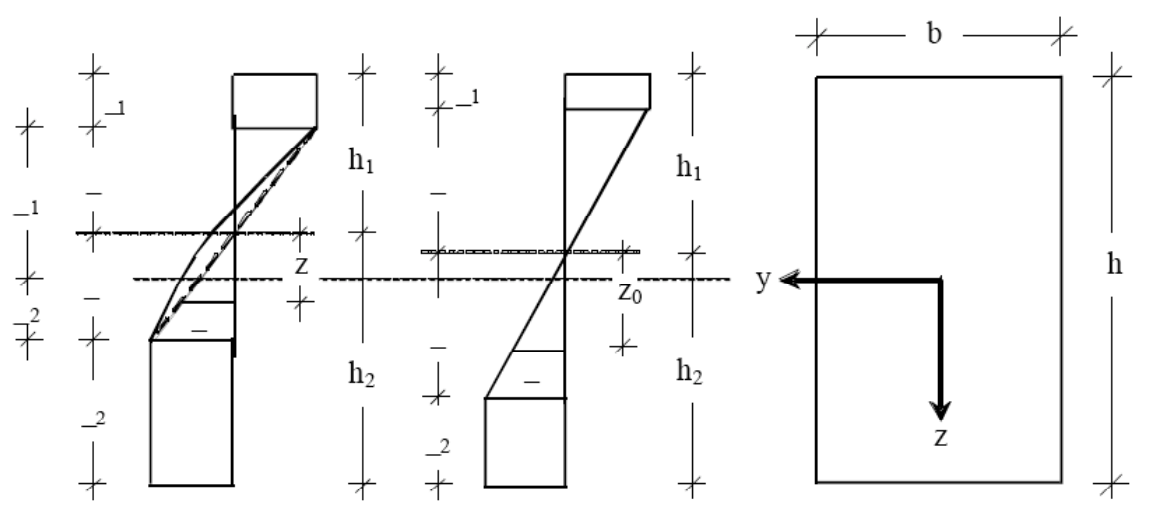

(a)

(b)

Fig. (12). Plastic regions and stress distributions in a solid cross-section. 
A displacement of the neutral line (in doubly symmetric cross-sections) takes place in the case of simultaneous action of a bending moment and an axial force and only in the elastic or elastoplastic region, while the form and position of the neutral line in the plastic region is not affected.

This displacement is significant and ranges (for the case studies presented herein) from $5 \%$ to $30 \%$ of the height of an I-cross-section and from $2 \%$ to $10 \%$ for a solid orthogonal one.

A phenomenon that appears in solid orthogonal crosssections is the evolution of the form of the neutral line which from a straight line becomes a curve.

The aforementioned alteration of the form and the displacement of the neutral line lead to the change of the corresponding resistant moments, which for the I-section of the case study amounts to $25 \%$ for the upper resistant moment and to $45 \%$ for the bottom one.

The corresponding change for the solid orthogonal crosssection studied herein amounts from 20 to $25 \%$.

\section{CONFLICT OF INTEREST}

The authors confirm that this article content has no conflicts of interest.

\section{ACKNOWLEDGEMENT}

Declared none.

\section{REFERENCES}

[1] Huber WA, Beedle SL. Residual stresses and Compressive Strength of Steel. Welding J 1954; 33: 589-614.

[2] Alpsten AG. Thermal Residual Stresses in Hot-Rolled Steel Members. Report No 337.3. USA: Fritz Laboratory 1968.

[3] Michaltsos GT. The elastoplastic analysis on Steel Structures. (in Greek). Athens: Symeon Publ., 2009.

[4] Marcelin JL, Abouaf M, Chenot JL. Analysis of Residual Stresses in Hot-Rolled Complex Beams. Comput Methods Appl Mech Eng 1968; 56(1): 1-16.

[5] Dixit US, Dixit PA. A study on residual stresses in rolling. Int J Mach Tools Manuf 1997; 37(6): 837-53.

[6] Michaltsos GT, Sophianopoulos DS. The effect of thermal residual stresses on the bearing capacity of Hot-Rolled I sections under Combined Bending and Axial Force. Proc. of the $6^{\text {th }}$ Nat Conf on Steel Structures, Patras, Greece, 2002.

[7] Sophianopoulos DS, Michaltsos GT. The effect of Thermal residual stresses on the bearing capacity of Hot-Rolled I sections under Combined Bending and Shearing Force. Proc. of the $3^{\text {rd }}$ European Conf on Steel Structures - EUROSTEEL 2002, Coibra, Portugal, 2002.

[8] Eurocode 3. Part 8. Thermal stresses. European Committee for Standardization, Brussels, 2003.

Received: April 30, 2013

Revised: June 12, 2013

Accepted: June 12, 2013

(C) Raftoyiannis and Michaltsos; Licensee Bentham Open.

This is an open access article licensed under the terms of the Creative Commons Attribution Non-Commercial License (http://creativecommons.org/licenses/by-nc/3.0/) which permits unrestricted, non-commercial use, distribution and reproduction in any medium, provided the work is properly cited. 